\title{
Inverse Relationship Between Serum Cystatin C and Sudomotor Function Detected by SUDOSCAN in Chinese Patients With Type 2 Diabetes Mellitus
}

\section{Qingyu Guo}

East Region Military Command General Hospital

Bin Lu

East Region Military Command General Hospital

\section{Liping Wang}

Central Hospital of Zi Bo

\section{Zhanhong Guo}

East Region Military Command General Hospital

\section{Wenjing Song}

Shanghai Sixth People's Hospital East

\section{Pu Zang}

East Region Military Command Genaral Hospital

\section{Ping Gu}

East Region Military Command General Hospital

\section{Ling Li}

Chinese Navy No.971. Hospital

Jiaqing Shao ( $\nabla$ shaojiaqing@nju.edu.cn )

East Region Military Command General Hospital

\section{Research}

Keywords: Cystatin C, Sudomotor function, SUDOSCAN, Electrochemical skin conductance, Diabetes mellitus, Type 2

Posted Date: December 16th, 2020

DOl: https://doi.org/10.21203/rs.3.rs-128671/v1

License: (c) (i) This work is licensed under a Creative Commons Attribution 4.0 International License.

Read Full License 


\section{Abstract}

Background and aims:To explore the relationship between serum cystatin $\mathrm{C}$ and sudomotor function produced by the SUDOSCAN in subjects with type 2 diabetes mellitus.

Methods:The research incorporated 917 Chinese individuals with type 2 diabetes mellitus from May 2017 to May 2019. All patients underwent SUDOSCAN and examined cystatin C. SUDOSCAN was evaluated with electrochemical skin conductance in hands (HESC) and feet (FESC), asymmetry ratio in hands (HASYM) and feet (FASYM). Average FESC $<60 \mu S$ was defined as sudomotor dysfunction. 917 patients were classified into 791 diabetic patients without sudomotor dysfunction, and 126 diabetic patients complicated with sudomotor dysfunction. The difference of cystatin $\mathrm{C}$ between two groups was compared firstly, the difference of SUDOSCAN metrics among tertiles of serum cystatin C was also compared. The spearman and multiple linear regression analyses were applied to analyze the association between serum cystatin $\mathrm{C}$ and SUDOSCAN metrics.

Results:The total prevalence of sudomotor dysfunction was $13.74 \%$. Patients with sudomotor dysfunction had a higher level of cystatin $\mathrm{C}(P<0.001)$. With increasing tertiles of serum cystatin $\mathrm{C}, \mathrm{ESC}$ declined, ASYM elevated, and the prevalence of sudomotor dysfunction increased $(P<0.001)$. The spearman analysis performed an association between serum cystatin $C$ and SUDOSCAN metrics including HESC $(r=-0.143, P<0.001)$, FESC $(r=-0.178, P<0.001)$, HASYM $(r=0.169, P<0.001)$, FASYM $(r=0.174, P<0.001)$. Multivariate regression linear analyses demonstrated cystatin $C$ was independently linked with HESC $(B=-14.657, P<0.001)$ and FESC $(B=-10.015, P=0.022)$ levels after controlling for potential confounders.

Conclusions:Serum cystatin $\mathrm{C}$ is inversely linked with sudomotor function detected by SUDOSCAN in Chinese patients with type 2 diabetes mellitus.

\section{Introduction}

Diabetic peripheral neuropathy (DPN) is the most common diabetic complications, which makes serious damage to the nerves in our arms, hands, legs, and feet. Previous study revealed that more than half of people with diabetes were complicated with DPN [1]. Distal symmetric polyneuropathy (DSPN) is one of the most shared types of DPN, which characterized by chronic, symmetrical, prolonged length and sensorimotor polyneuropathy [2]. DSPN is a significant risk element for diabetic foot, so it can greatly increase the mortality and disability of subjects with type 2 diabetes mellitus (T2DM) [3]. It often affects distal diminutive nerve fibers and manifests as aching neuropathy, however, upon most occasions, symptoms of DSPN are insidious, up to $50 \%$ of patients without evident symptoms in the early stage of DSPN, symptoms are severe when they are diagnosed [4]. At present, the most trustworthy diagnosis of DPN is the biopsy, but it is difficult to carry out on a large scale because it is an invasive operation [5].

SUDOSCAN (Impeto Medical, Paris, France) is a new screening technique for the detection of DSPN through assessment of sweat gland sudomotor function as a reflection of early peripheral small fiber 
neuropathy impairment [6]. As a simple and impersonal screening technique for DSPN, SUDOSCAN has been popular in Caucasian and Indian people [7]. The measurement of sudomotor function including electrochemical skin conductance (ESC, measured in $\mu S$ ). And the basic principle of detecting ESC is reaction between sweat chlorides and electrodes. Results are presented as HESC, FESC, symmetry ratio in hands (HASYM) and feet (FASYM) [8]. Sudomotor dysfunction can be detected by SUDOSCAN in the inchoate stage of DSPN. Compared with other detection methods, the ESC detection is more sensitive, hence more and more doctors are prone to use it for the inchoate diagnosis of DPN [9].

Cystatin C (Cys-C), as a small molecular substance, is an endogenous cysteine protease inhibitor. It has been used as a sensitive metric to evaluate renal function because of free filtration in glomeruli [10]. In the last few decades, some researchers have found serum Cys-C level was positively associated with the mortality of cardiovascular and cerebrovascular diseases like coronary heart disease, peripheral arterial disease, stroke and so on, which explains its roles beyond information about renal function [11]. In addition, serum Cys-C level was positively linked with the prevalence of central and peripheral neuropathy in T2DM [12, 13].

However, the link between serum Cys-C and sudomotor function was not explored clearly yet. In this study, we aim to explore the correlation between serum Cys-C and sudomotor function assessed by SUDOSCAN in Chinese subjects with T2DM.

\section{Methods}

\section{Participants}

The research enrolled 917 T2DM inpatients at the endocrinology department of the General Hospital of Eastern Theater Command from May 2017 to May 2019. The whole of them conformed to the 1999 WHO diagnostic criteria for diabetes [14]. Exclusion criteria included: 1. Individuals with severe illness or acute stress such as heart failure, liver failure, acute or chronic inflammatory disorders, malignant disease and surgery. 2. Subjects with abnormal renal function (eGFR $<60 \mathrm{ml} / \mathrm{min} / 1.73 \mathrm{~m} 2$ ). 3. Subjects who had a history of using oral medications that may affect the nervous system. 4 . Subjects with the rest of metabolic disorder like the lack of vitamin B12. The research was supported by the local ethics committee.

\section{Anthropometric indices and laboratory examination}

Medical history including age, gender, diabetes duration, height, weight, smoking was gained through medical records. Systolic blood pressure (SBP), diastolic blood pressure (DBP) were measured, body mass index (BMI) was calculated. Blood and urine samples were gathered overnight fasting for hemoglobin $A_{1} C(H b A 1 c)$, uric acid (UA), total bilirubin (T-BIL), direct bilirubin, (D-BIL), triglyceride (TG), total cholesterol (TC), high density lipoprotein (HDL), low density lipoprotein (LDL), fasting blood glucose (FBG), 2-hour postprandial blood glucose (PBG), serum urea nitrogen (BUN), cystatin C (Cys-C), free fat acid (FFA), homocysteine (Hcy) and serum creatinine (SCr). eGFR was counted using Chronic Kidney 
Disease- Epidemiology Collaboration(CKD-EPI) equation [15]. Serum Cys-C level was measured by immunoturbidimetry.

\section{SUDOSCAN test}

SUDOSCAN (Impeto Medical, Paris, France) as a quick, nonintrusive and quantitative device, was applied to assess sudomotor function on the basis of sweat chloride concentrations. The appliance had two parts of electrodes which are connected to a computer. Patients placed their limbs on the electrodes only about two or three minutes and an incremental low voltage $(<4 \mathrm{~V})$ was applied to the electrodes automatically. Quantificational data were showed as HESC, FESC, HASYM and FASYM. Sudomotor dysfunction refers to average FESC is $<60 \mu \mathrm{S}$. 917 patients were classified into 791 diabetic patients without sudomotor dysfunction, and 126 diabetic patients complicated with sudomotor dysfunction. The operation is painless, rapid and no specific preparation is needed. What's more, the quantitative results are unaffected by room temperature [16].

\section{Statistical analysis}

SPSS 22.0 software was applied for statistical analyses. All data were presented as the mean \pm standard deviation (SD), median (upper and lower quartiles) or proportions according to different data types. For continuous variables, Student's t-test was used to compare two groups, and one way ANOVA was applied to contrast more than two samples. Wilcoxon rank-sum test was applied for abnormal distributions. Chisquare test was used for categorical variables. Spearman's rank correlation was performed to evaluate the association of serum Cys-C and SUDOSCAN metrics. And multiple linear regression analysis was carried out to explore the link between serum Cys-C and SUDOSCAN metrics after adjusting for age, diabetes duration, Cys-C, SBP, DBP, BMI, HbA1C, TC, HDL, FFA, Scr and eGFR. $P<0.05$ was considered statistically significant.

\section{Results}

Clinical characteristics of individuals with and without sudomotor dysfunction.

The whole of baseline information was shown in Table 1. The mean age of all participants was $54.76 \pm 12.42$ years, and the median duration of diabetes was $7(2,11)$ years. The total prevalence of sudomotor dysfunction was $13.74 \%$. Compared without sudomotor dysfunction group, patients with sudomotor dysfunction were older and had a longer duration of diabetes $(P<0.001)$. Besides, patients with sudomotor dysfunction showed increased levels of BUN, UA and Cys-C, and lower levels of eGFR and FFA $(P<0.05)$.

Clinical characteristics and SUDOSCAN parameters of subjects among tertiles (T1-T3) of serum Cys-C level.

Farther statistics after dividing participants into groups on the basis of tertiles for serum Cys-C level. Table 2 showed the following points. First of all, participants with the highest tertiles of serum Cys-C were 
older and had a longer duration of diabetes $(P<0.001)$. What's more, patients with the highest tertiles of serum Cys-C had higher SBP, BUN, SCr, UA and Hcy, lower eGFR, TC, FBG and $\mathrm{HbA} 1 \mathrm{C}(P<0.05)$. Of note, patients with the highest tertiles of serum Cys-C had higher HASYM, FASYM and lower hand ESC, foot $\operatorname{ESC}(P<0.001)$.

Prevalence of sudomotor dysfunction in different tertiles of serum Cys-C level.

Patients were divided into three groups according to the tertiles of serum Cys-C level, the prevalence of sudomotor dysfunction increased with the increase of Cys-C. $(P<0.001)$ (Figure 1).

The association between clinical characteristics and metrics of SUDOSCAN.

The association between serum Cys-C and metrics of SUDOSCAN was constructed with Spearman's rank correlation analysis. Serum Cys-C level negatively associated with hand and foot ESC $(P<0.001)$. What's more, serum Cys-C level positively associated with HASYM and FASYM $(P<0.001)$. Age, diabetes duration and SBP inversely associated with hand and foot ESC, positively associated with HASYM and FASYM $(P<0.05)$. eGFR positively associated with hand and foot ESC, inversely associated with HASYM and FASYM $(P<0.05)$.BMI only positively correlated with hand $\operatorname{ESC}(P<0.05)$. Of note, there was no relationship between $\mathrm{HbA} 1 \mathrm{C}$ and parameters of SUDOSCAN.(Table 3)

Multivariate regression linear analyses of association between serum Cys-C and parameters of SUDOSCAN.

Multivariate regression linear analyses were adjusted for age, diabetes duration, Cys-C, SBP, DBP, BMI, $\mathrm{HbA1C}, \mathrm{TC}, \mathrm{HDL}, \mathrm{FFA}$, Scr and eGFR. The data demonstrated cystatin $\mathrm{C}$ was independently linked with HESC and FESC levels. But the link was disappeared for HASYM or FASYM. Besides, for FESC, the older age and the longer diabetes duration were risk factors, the higher BMI, HDL and eGFR were protected factors. For HASYM, elevated SBP was a dangerous factor, rather DBP was a protect factor. Finally, for FASYM, elevated BMI and TC were protect factors. (Table 4)

\section{Discussion}

Overall, our study revealed serum Cys-C inversely associated with sudomotor function, which detected by SUDOSCAN. Patients with sudomotor dysfunction had a higher level of cystatin $C(P<0.001)$. With increasing tertiles of serum cystatin C, ESC declined, ASYM elevated, and the prevalence of sudomotor dysfunction increased $(P<0.001)$.

As one of the most vital risk factors for diabetic foot, DSPN can greatly increase the mortality and disability of individuals with T2DM [3]. The main manifestation of DSPN is the destruction of small unmyelinated sympathetic nerve fibers, so it is difficult to detect early DSPN. The traditional and common methods to explore small fiber neuropathies including skin biopsy, quantitative sudomotor axon reflex test (QSART) and neuropad [2]. The most trustworthy diagnosis of DPN is the biopsy, but it is difficult to carry out on a large scale, because it is an invasive operation [17]. QSART is a recommended method, but 
it takes more time. Also, neuropad is not a quantitative test, and it has low sensitivity and specificity [2]. ESC measurement detecting by SUSOSCAN is an emerging method in recent years [18]. American Association of Clinical Endocrinologists (AACE) guidelines in 2015 mentioned sudomotor function can be applied to assess early neuropathy [19]. Sudomotor function test can be used in a wide range because it is fast, impersonal quantitative and not complicated.

Previous studies found longer diabetes duration, older age, higher $\mathrm{HbA} 1 \mathrm{c}$ levels had a dangerous effect on the incidence of DPN [20]. Consistent with these, our study also found age and diabetes duration were negatively associated with FESC. But there was no relationship between $\mathrm{HbA1c}$ and any metrics of SUSOSCAN, we speculated that glycemic variability may play a greater role in the sudomotor dysfunction, exactly as a recent study demonstrated glycemic variability metric like time in range play a greater role in the prevalence of diabetic cardiovascular autonomic neuropathy [21]. What's more, as small sympathetic unmyelinated C-fibers innervate sweat glands, we found SBP negatively associated with ESC, positively correlated with HASYM and FASYM. So the older people who are easy to suffer from a higher level of SBP, should pay more attention to the examination of Cys-C level and sudomotor function. Finally, a mild relationship between BMI and sudomotor dysfunction was found. However, most researchers have found $\mathrm{BMI}$ had a dangerous effect on DPN. However, Xu et al hold a diverse view: lower BMI might be a latent risk factor for DPN [22]. Also, a new research from China in which they reported that lower BMI had a dangerous effect on diabetic complications [23]. In the multivariate regression linear analyses, we also found TC was negative with HASYM and FASYM, this is probably because lower TC may indicated that nutrition in patients with DPN was imbalance, which may negatively affected the rehabilitation of DPN [22].

Cys- $C$, as a sensitive metric to evaluate renal function because of free filtration in glomeruli, may provide more information about diabetes mellitus beyond renal function [24]. In the last few decades, some researchers have found positive associations between serum Cys-C level and the risk for T2DM [25]. Qian et al confirmed the level of serum Cys-C was positively linked with retinopathy in characters of T2DM [26]. Qamar et al found that serum Cys-C level could be applied as an inchoate evaluation for the renal dysfunction in T2DM individuals with normal urine protein [27]. Zhao et al demonstrated serum CysC linked with a high prevalence of diabetic foot ulceration in T2DM [28]. Also, there were studies research the link between Cys-C and diabetic neuropathy. One study found serum Cys-C level was positively linked with cardiovascular autonomic neuropathy in subjects with T2DM [29]. Hu et al demonstrated high Cys-C concentration was closely linked with DPN and the cutoff value of serum Cys-C indicating DPN was $1.25 \mathrm{mg} / \mathrm{L}$ in men and $1.05 \mathrm{mg} / \mathrm{L}$ in women [30]. Different researchers have also proven that Cys-C has a significant effect on central and peripheral nervous diseases [31,32]. In the central nervous system, Cys-C functioned as an endogenous cysteine protease inhibitor. It has been testified Cys-C plays a regulatory role on agglomeration and sedimentation of amyloid $\triangle$ [33]. Compared to normal people, subjects had higher serum Cys-C, who suffered from mild cognitive impairment, Alzheimer's or Parkinson's [34-36]. In addition, the CysC level in cerebrospinal fluid varied significantly in demyelinating diseases such as Guillain Barre Syndrome [37]. Nagai et al also demonstrated Cys-C was linked with the apoptosis of 
neurons [38]. Consistent with these studies, our study showed a robust link between serum Cys-C level and sudomotor dysfunction in Chinese T2DM. Subjects with sudomotor dysfunction had a higher level of cystatin C. With increasing tertiles of serum cystatin C, ESC declined, ASYM elevated, and the prevalence of sudomotor dysfunction increased. More importantly, serum Cys-C inversely linked with sudomotor function like FESC and HESC. Serum Cys-C level was bound up with eGFR, and there was a higher level of eGFR in individuals complicated sudomotor dysfunction, eGFR positively linked with ESC and inversely linked with ASYM. Nevertheless, after adjusting for age, diabetes duration, Cys-C, SBP, DBP, BMI, HbA1C, TC, HDL, FFA, Scr, the relationship between eGFR and HESC disappeared.

At present, the destructive mechanism between serum Cys-C and sudomotor dysfunction is not fully clarified, which may include the following points. Cys-C may participate in the pathological process of diabetic microvascular complications through inflammation, oxidative stress, degradation of extracellular matrix and vascular remodeling [39]. Cys-C and its degradation products can activate neutrophils, thus mediating inflammatory response, and increasing the concentration of C-reactive protein. A high concentration of C-reactive protein can lead to dysfunction of vascular endothelial or destruction of endothelial cells directly [40]. Besides, Cys-C can inhibit homocysteine catabolase, the high level of homocysteine can lead to oxidative stress, destruction of vascular endothelial cells [41].

Serum Cys-C measurement is simple and routine, and is highly accessible in every community hospital. Therefore, serum Cys-C level can be applied easily as one of the risk factors for sudomotor dysfunction. Careful consideration of SUDOSCAN screening is needed when managing subjects with T2DM and a high level of serum Cys-C concentration.

Nevertheless, our study has a few limitations. First of all, our study only enrolled individuals with T2DM, so it was not possible to explore the relationship between other types of diabetes and sudomotor function. Secondly, this was a cross-sectional study, so it was impossible to explore the causality between serum Cys-C and sudomotor dysfunction.

\section{Conclusion}

In conclusion, our data showed that serum Cys-C inversely linked with sudomotor function like FESC and HESC, which were detected by SUDOSCAN. Besides, serum Cys-C level positively associated with HASYM and FASYM. Further prospective research is needed to identify the risk role of serum Cys-C for sudomotor dysfunction in subjects with T2DM.

\section{Declarations}

Acknowledgements: We thank all the clinicians enrolled in this study.

Author Contributions: Qingyu Guo, Bin Lu, Liping Wang and Zhanhong Guo conceived and designed the research; Qingyu Guo and Wenjing Song analyzed and interpreted the data; Qingyu Guo, Pu Zang and Ping Gu performed the statistical analysis; Qingyu Guo wrote the manuscript; Ling Li and Jiaqing Shao 
critically revised the manuscript for key intellectual content. Qingyu Guo is responsible for the integrity of the work. All authors read and approved the final manuscript.

Funding: We thank all the clinicians enrolled in this study. This work was supported by the National Natural Science Foundation of China (81774134, 81873174); Natural Science Foundation of Jiangsu Province of China (BK20150558, BK20171331); Postdoctoral Foundation of Jiangsu Province of China (1501120C); Young Medical Key Talents Project of Jiangsu Province(QNRC2016902); Jiangsu Province 333 Talent Funding Project (BRA2017595).

Availability of data and materials: The datasets generated during and/or analyzed during the current study are available from the corresponding author and the senior author on reasonable request.

Ethics approval and consent to participate: The study was approved by the Ethics Committee of Jinling Hospital, Nanjing University and was performed according to the Declaration of Helsinki.

Consent for publication: Not applicable.

Competing interests:The authors have nothing to disclose.

\section{Author details:}

1 Jinling Hosp Dept Endocrinology, Nanjing Univ, Sch Med, Nanjing, China.

${ }^{2}$ Central Hospital of Zi Bo, Shandong, China.

3 Jinling Hosp Dept Endocrinology, Nanjing Med Univ, Nanjing, China.

${ }^{4}$ Shanghai Sixth People's Hospital East, Shanghai, China.

${ }^{5}$ Chinese Navy No.971.Hospital, Shandong, China.

\section{${ }^{*}$ Correspondence author:}

Ling Li, MD

Department of Endocrinology, Chinese Navy No.971.Hospital, Shandong, 266000, P R China

22 Minjiang Road, Qingdao, Shandong, 266000, China

Tel: 18561898066

E-mail address: 897426605@qq.com

Jia-qing Shao, MD, PhD.

Jinling Hosp Dept Endocrinology, Nanjing Univ, Sch Med, Nanjing, 210002, P R China 
305 East Zhongshan Road, Nanjing, Jiangsu, 210002, China

Tel: +86-25-80860354

E-mail address: shaojiaqing@nju.edu.cn

\section{References}

1. Vinik A, Ullal J, Parson H K, et al. Diabetic neuropathies: clinical manifestations and current treatment options. Nat Clin Pract Endocrinol Metab, 2006; 2:269-281.

2. Callaghan $B C$, Cheng $H T$, Stables $C L$, et al. Diabetic neuropathy: clinical manifestations and current treatments. Lancet Neurol, 2012; 11:521-534.

3. Wu S C, Driver V R, Wrobel J S, et al. Foot ulcers in the diabetic patient, prevention and treatment. Vasc Health Risk Manag, 2007; 3:65-76.

4. Rage M, Van Acker N, Knaapen M W, et al. Asymptomatic small fiber neuropathy in diabetes mellitus: investigations with intraepidermal nerve fiber density, quantitative sensory testing and laserevoked potentials. J Neurol, 2011; 258:1852-1864.

5. Callaghan B C, Cheng H T, Stables C L, et al. Diabetic neuropathy: clinical manifestations and current treatments. Lancet Neurol, 2012; 11:521-534.

6. Vinik A I, Nevoret M L, Casellini C. The New Age of Sudomotor Function Testing: A Sensitive and Specific Biomarker for Diagnosis, Estimation of Severity, Monitoring Progression, and Regression in Response to Intervention. Front Endocrinol (Lausanne), 2015; 6:94.

7. Yajnik C S, Kantikar V V, Pande A J, et al. Quick and simple evaluation of sudomotor function for screening of diabetic neuropathy. ISRN Endocrinol, 2012; 2012:103714.

8. Smith A G, Lessard M, Reyna S, et al. The diagnostic utility of Sudoscan for distal symmetric peripheral neuropathy. J Diabetes Complications, 2014; 28:511-516.

9. Novak P. Electrochemical Skin Conductance Correlates with Skin Nerve Fiber Density. Front Aging Neurosci, 2016; 8:199.

10. Mussap $M$, Dalla V M, Fioretto $P$, et al. Cystatin $C$ is a more sensitive marker than creatinine for the estimation of GFR in type 2 diabetic patients. Kidney Int, 2002; 61:1453-1461.

11. Svensson-Farbom P, Ohlson A M, Almgren P, et al. Cystatin C identifies cardiovascular risk better than creatinine-based estimates of glomerular filtration in middle-aged individuals without a history of cardiovascular disease. J Intern Med, 2014; 275:506-521.

12. Chung J O, Cho D H, Chung D J, et al. Serum Cystatin C Levels are Positively Associated with Cardiovascular Autonomic Neuropathy in Patients with Type 2 Diabetes. Exp Clin Endocrinol Diabetes, 2015; 123:627-631.

13. Hu Y, Liu F, Shen J, et al. Association between serum cystatin C and diabetic peripheral neuropathy: a cross-sectional study of a Chinese type 2 diabetic population. Eur J Endocrinol, 2014; 171:641-648. 
14. Alberti K G, Zimmet P Z. Definition, diagnosis and classification of diabetes mellitus and its complications. Part 1: diagnosis and classification of diabetes mellitus provisional report of a WHO consultation. Diabet Med, 1998; 15:539-553.

15. Sudchada P, Laehn S. Comparisons of GFR estimation using the CKD Epidemiology Collaboration (CKD-EPI) equation and other creatinine-based equations in Asian population: a systematic review. Int Urol Nephrol, 2016; 48:1511-1517.

16. Vinik A I, Nevoret M L, Casellini C. The New Age of Sudomotor Function Testing: A Sensitive and Specific Biomarker for Diagnosis, Estimation of Severity, Monitoring Progression, and Regression in Response to Intervention. Front Endocrinol (Lausanne), 2015; 6:94.

17. Lauria G, Devigili G. Skin biopsy as a diagnostic tool in peripheral neuropathy. Nat Clin Pract Neurol, 2007; 3:546-557.

18. Casellini C M, Parson H K, Richardson M S, et al. Sudoscan, a noninvasive tool for detecting diabetic small fiber neuropathy and autonomic dysfunction. Diabetes Technol Ther, 2013; 15:948-953.

19. Handelsman Y, Bloomgarden Z T, Grunberger G, et al. American association of clinical endocrinologists and american college of endocrinology - clinical practice guidelines for developing a diabetes mellitus comprehensive care plan - 2015. Endocr Pract, 2015; 21 Suppl 1:1-87.

20. Adler A I, Boyko E J, Ahroni J H, et al. Risk factors for diabetic peripheral sensory neuropathy. Results of the Seattle Prospective Diabetic Foot Study. Diabetes Care, 1997; 20:1162-1167.

21. Guo Q, Zang P, Xu S, et al. Time in Range, as a Novel Metric of Glycemic Control, Is Reversely Associated with Presence of Diabetic Cardiovascular Autonomic Neuropathy Independent of HbA1c in Chinese Type 2 Diabetes. J Diabetes Res, 2020; 2020:5817074.

22. Xu F, Zhao L H, Su J B, et al. The relationship between glycemic variability and diabetic peripheral neuropathy in type 2 diabetes with well-controlled HbA1c. Diabetol Metab Syndr, 2014; 6:139.

23. Zhang $Y$, Guo $Y$, Shen $X$, et al. Lower body mass index is not of more benefit for diabetic complications. J Diabetes Investig, 2019; 10:1307-1317.

24. Mussap $M$, Dalla V M, Fioretto $P$, et al. Cystatin $C$ is a more sensitive marker than creatinine for the estimation of GFR in type 2 diabetic patients. Kidney Int, 2002; 61:1453-1461.

25. Sahakyan K, Lee K E, Shankar A, et al. Serum cystatin C and the incidence of type 2 diabetes mellitus. Diabetologia, 2011; 54:1335-1340.

26. Qian C, Wan G M, Yan P S, et al. Correlation between Cystatin C and retinopathy of type-two diabetes mellitus patients. J Biol Regul Homeost Agents, 2017; 31:99-103.

27. Qamar A, Hayat A, Ahmad T M, et al. Serum Cystatin C as an Early Diagnostic Biomarker of Diabetic Kidney Disease in Type 2 Diabetic Patients. J Coll Physicians Surg Pak, 2018; 28:288-291.

28. Zhao J, Deng W, Zhang Y, et al. Association between Serum Cystatin C and Diabetic Foot Ulceration in Patients with Type 2 Diabetes: A Cross-Sectional Study. J Diabetes Res, 2016; 2016:8029340.

29. Chung J O, Cho D H, Chung D J, et al. Serum Cystatin C Levels are Positively Associated with Cardiovascular Autonomic Neuropathy in Patients with Type 2 Diabetes. Exp Clin Endocrinol 
Diabetes, 2015; 123:627-631.

30. Hu Y, Liu F, Shen J, et al. Association between serum cystatin C and diabetic peripheral neuropathy: a cross-sectional study of a Chinese type 2 diabetic population. Eur J Endocrinol, 2014; 171:641-648.

31. Deng A, Irizarry M C, Nitsch R M, et al. Elevation of cystatin C in susceptible neurons in Alzheimer's disease. Am J Pathol, 2001; 159:1061-1068.

32. Nakashima I, Fujinoki M, Fujihara K, et al. Alteration of cystatin $\mathrm{C}$ in the cerebrospinal fluid of multiple sclerosis. Ann Neurol, 2007; 62:197-200, 205.

33. Mi W, Pawlik M, Sastre M, et al. Cystatin C inhibits amyloid-beta deposition in Alzheimer's disease mouse models. Nat Genet, 2007; 39:1440-1442.

34. Wang R, Chen Z, Fu Y, et al. Plasma Cystatin C and High-Density Lipoprotein Are Important Biomarkers of Alzheimer's Disease and Vascular Dementia: A Cross-Sectional Study. Front Aging Neurosci, 2017; 9:26.

35. Dong X, Zheng D, Nao J. Measurement of serum cystatin C: A valuable tool for evaluating dyskinesia in Parkinson's disease. Neurosci Lett, 2019; 705:172-176.

36. Slinin Y, Peters K W, Ishani A, et al. Cystatin $C$ and cognitive impairment 10 years later in older women. J Gerontol A Biol Sci Med Sci, 2015; 70:771-778.

37. Bollengier F. Cystatin C, alias post-gamma-globulin: a marker for multiple sclerosis? J Clin Chem Clin Biochem, 1987; 25:589-593.

38. Nagai A, Ryu J K, Terashima M, et al. Neuronal cell death induced by cystatin $C$ in vivo and in cultured human CNS neurons is inhibited with cathepsin B. Brain Res, 2005; 1066:120-128.

39. Kaneko R, Sawada S, Tokita A, et al. Serum cystatin C level is associated with carotid arterial wall elasticity in subjects with type 2 diabetes mellitus: A potential marker of early-stage atherosclerosis. Diabetes Res Clin Pract, 2018; 139:43-51.

40. Shlipak M G, Katz R, Cushman M, et al. Cystatin-C and inflammatory markers in the ambulatory elderly. Am J Med, 2005; 118:1416.

41. Deng A, Irizarry M C, Nitsch R M, et al. Elevation of cystatin C in susceptible neurons in Alzheimer's disease. Am J Pathol, 2001; 159:1061-1068.

\section{Tables}

Table 1 Clinical characteristics of individuals with and without sudomotor dysfunction. 


\begin{tabular}{|c|c|c|c|c|}
\hline & \multicolumn{4}{|c|}{ Sudomotor dysfunction } \\
\hline & No & Yes & $x^{2} / t / z$ & $P$ \\
\hline $\mathrm{N}$ & 791 & 126 & - & - \\
\hline Male $(n, \%)$ & $533(67.38)$ & $83(65.87)$ & 0.11 & 0.737 \\
\hline Age $(y)$ & $54.13 \pm 12.25$ & $58.74 \pm 12.78$ & -3.90 & $<0.001$ \\
\hline Diabetes duration $(\mathrm{y})$ & $6(2,11)$ & $10(5,15)$ & -4.74 & $<0.001$ \\
\hline Smoking (n, \%) & $252(31.86)$ & $35(27.78)$ & 0.84 & 0.359 \\
\hline $\mathrm{SBP}(\mathrm{mmHg})$ & $131.80 \pm 15.52$ & $133.90 \pm 19.52$ & -1.15 & 0.253 \\
\hline $\mathrm{DBP}(\mathrm{mmHg})$ & $79.24 \pm 9.69$ & $78.06 \pm 9.30$ & 1.27 & 0.206 \\
\hline T-BIL $(\mu \mathrm{mol} / \mathrm{L})$ & $10.50(7.65,13.95)$ & $9.40(7.65,14.2)$ & -0.60 & 0.546 \\
\hline $\mathrm{D}-\mathrm{BIL}(\mu \mathrm{mol} / \mathrm{L})$ & $3.10(2.30,4.40)$ & $3.40(2.35,4.95)$ & -0.44 & 0.658 \\
\hline $\mathrm{BUN}(\mathrm{mmol} / \mathrm{L})$ & $5.63 \pm 1.53$ & $5.98 \pm 1.57$ & -2.37 & 0.018 \\
\hline $\operatorname{SCr}(\mu \mathrm{mol} / \mathrm{L})$ & $56.00(47.00,67.00)$ & $58.00(48.00,69.50)$ & -1.88 & 0.061 \\
\hline eGFR $\left(\mathrm{ml} / \mathrm{min} / 1.73 \mathrm{~m}^{2}\right)$ & $107.56 \pm 17.67$ & $100.79 \pm 16.82$ & 4.02 & $<0.001$ \\
\hline $\mathrm{UA}(\mu \mathrm{mol} / \mathrm{L})$ & $323.42 \pm 89.94$ & $343.71 \pm 94.91$ & -2.33 & 0.020 \\
\hline Cys-C (mg/L) & $1.00(0.87,1.18)$ & $1.17(0.99,1.37)$ & -5.77 & $<0.001$ \\
\hline $\mathrm{TC}(\mathrm{mmol} / \mathrm{L})$ & $4.44(3.81,5.13)$ & $4.41(3.58,5.24)$ & -0.51 & 0.609 \\
\hline $\mathrm{TG}(\mathrm{mmol} / \mathrm{L})$ & $1.55(1.04,2.49)$ & $1.50(1.12,2.24)$ & -0.37 & 0.713 \\
\hline $\mathrm{HDL}(\mathrm{mmol} / \mathrm{L})$ & $1.03(0.89,1.24)$ & $1.07(0.92,1.30)$ & -1.30 & 0.195 \\
\hline $\mathrm{LDL}(\mathrm{mmol} / \mathrm{L})$ & $2.60(2.04,3.26)$ & $2.67(2.03,3.30)$ & -0.03 & 0.974 \\
\hline Hcy $(\mu \mathrm{mol} / \mathrm{L})$ & $10.70(8.90,12.50)$ & $11.30(10.10,13.30)$ & -1.50 & 0.134 \\
\hline $\mathrm{FFA}(\mathrm{mmol} / \mathrm{L})$ & $0.46(0.34,0.62)$ & $0.34(0.27,0.47)$ & -3.18 & 0.001 \\
\hline $\mathrm{FBG}(\mathrm{mmol} / \mathrm{L})$ & $7.44 \pm 2.52$ & $7.83 \pm 2.93$ & -1.15 & 0.249 \\
\hline $\mathrm{PBG}(\mathrm{mmol} / \mathrm{L})$ & $15.63 \pm 3.76$ & $16.27 \pm 4.07$ & -1.71 & 0.089 \\
\hline $\mathrm{HbA} 1 \mathrm{C}(\%)$ & $8.73 \pm 2.16$ & $8.72 \pm 2.15$ & 0.033 & 0.974 \\
\hline $\mathrm{BMI}(\mathrm{kg} / \mathrm{m} 2)$ & $25.48 \pm 3.67$ & $24.90 \pm 4.34$ & 1.61 & 0.107 \\
\hline
\end{tabular}

a SBP, systolic blood pressure, DBP, diastolic blood pressure, T-BIL, total bilirubin, D-BIL, direct bilirubin, BUN, blood urea nitrogen, $\mathrm{SCr}$, serum creatinine, eGFR, estimated glomerular filtration rate, UA, uric acid, Cys-C, cystatin C, TC, total cholesterol, TG, triglyceride, HDL, high density lipoprotein, LDL, low density 
lipoprotein, Hcy, homocysteine, FFA, free fat acid, FBG, fasting blood glucose, PBG, postprandial blood glucose, $\mathrm{HbA} 1 \mathrm{C}$, hemoglobin $\mathrm{A}_{1} \mathrm{C}, \mathrm{BMI}$, body mass index.

${ }^{\mathrm{b}}$ Normally distributed values in the table are presented as the means $\pm S D$, non-normally distributed values are presented as medians ( $25 \%$ and $75 \%$ interquartiles) and categorical variables are presented as frequencies (percentages). Student's t-test for comparison of two samples with a normal distribution, Wilcoxon rank-sum test for abnormal distributions.X2-test for categorical variables.

Table 2 Clinical characteristics and SUDOSCAN parameters of individuals among tertiles (T1-T3) of serum Cys-C level. 


\begin{tabular}{|c|c|c|c|c|c|}
\hline & \multicolumn{5}{|c|}{ Tertiles (T1-T3) of serum Cys-C } \\
\hline & $\mathrm{T} 1(<0.92 \mathrm{mg} / \mathrm{L})$ & $\begin{array}{l}\mathrm{T} 2(0.92-1.12 \\
\mathrm{mg} / \mathrm{L})\end{array}$ & $\mathrm{T} 3(>1.12 \mathrm{mg} / \mathrm{L})$ & $x 2 / F / z$ & $\mathrm{P}$ \\
\hline $\mathrm{N}$ & 312 & 302 & 303 & - & - \\
\hline Male (n, \%) & $195(62.50)$ & 215(71.19) & $206(67.99)$ & 5.39 & 0.067 \\
\hline Age $(y)$ & $49.00 \pm 11.89$ & $56.65 \pm 11.43$ & $60.79 \pm 11.03$ & 81.33 & $<0.001$ \\
\hline $\begin{array}{l}\text { Diabetes } \\
\text { duration (y) }\end{array}$ & $5(2,10)$ & $6(2,11)$ & $10(4,15)$ & 40.75 & $<0.001$ \\
\hline $\begin{array}{l}\text { Smoking (n, } \\
\%)\end{array}$ & $89(28.53)$ & $95(31.46)$ & 103(33.99) & 2.14 & 0.343 \\
\hline $\mathrm{SBP}(\mathrm{mmHg})$ & $129.23 \pm 13.39$ & $131.69 \pm 15.44$ & $135.44 \pm 18.66$ & 11.76 & $<0.001$ \\
\hline $\mathrm{DBP}(\mathrm{mmHg})$ & $78.90 \pm 9.51$ & $79.27 \pm 9.61$ & $79.07 \pm 9.83$ & 0.12 & 0.891 \\
\hline $\begin{array}{l}\text { T-BIL } \\
(\mu \mathrm{mol} / \mathrm{L})\end{array}$ & $10.80(8.10,14.80)$ & $10.00(8.00,13.80)$ & $9.75(7.30,13.25)$ & 4.99 & 0.083 \\
\hline $\begin{array}{l}\text { D-BIL } \\
(\mu \mathrm{mol} / \mathrm{L})\end{array}$ & $3.30(2.20,4.40)$ & $3.20(2.40,4.60)$ & $3.10(2.30,4.40)$ & 0.16 & 0.92 \\
\hline $\begin{array}{l}\text { BUN } \\
(\mathrm{mmol} / \mathrm{L})\end{array}$ & $5.15 \pm 1.34$ & $5.38 \pm 1.25$ & $6.51 \pm 1.65$ & 80.09 & $<0.001$ \\
\hline $\mathrm{SCr}(\mu \mathrm{mol} / \mathrm{L})$ & $49.00(41.00,57.00)$ & $55.00(48.00,64.00)$ & $68.00(58.00,81.00)$ & 246.21 & $<0.001$ \\
\hline $\begin{array}{l}\text { eGFR } \\
(\mathrm{ml} / \mathrm{min} / 1.73 \\
\left.\mathrm{m}^{2}\right)\end{array}$ & $118.56 \pm 14.15$ & $108.93 \pm 11.61$ & $92.06 \pm 15.64$ & 285.22 & $<0.001$ \\
\hline $\mathrm{UA}(\mu \mathrm{mol} / \mathrm{L})$ & $306.59 \pm 86.55$ & $321.73 \pm 92.67$ & $350.96 \pm 87.92$ & 19.62 & $<0.001$ \\
\hline TC (mmol/L) & $4.54(3.96,5.30)$ & $4.58(3.88,5.22)$ & $4.21(3.48,4.87)$ & 25.61 & $<0.001$ \\
\hline TG (mmol/L) & $1.62(1.11,2.67)$ & $1.46(0.95,2.44)$ & $1.53(1.10,2.22)$ & 3.68 & 0.159 \\
\hline $\begin{array}{l}\mathrm{HDL} \\
(\mathrm{mmol} / \mathrm{L})\end{array}$ & $1.05(0.91,1.24)$ & $1.07(0.91,1.26)$ & $1.02(0.88,1.20)$ & 7.62 & 0.022 \\
\hline $\begin{array}{l}\text { LDL } \\
(\mathrm{mmol} / \mathrm{L})\end{array}$ & $2.67(2.15,3.30)$ & $2.76(2.19,3.31)$ & $2.40(1.81,3.10)$ & 18.66 & $<0.001$ \\
\hline Hcy $(\mu \mathrm{mol} / \mathrm{L})$ & $9.45(7.95,11.13)$ & $10.70(9.10,11.90)$ & $12.60(10.90,15.80)$ & 78.49 & $<0.001$ \\
\hline $\begin{array}{l}\text { FFA } \\
\text { (mmol/L) }\end{array}$ & $0.48(0.36,0.60)$ & $0.42(0.30,0.61)$ & $0.45(0.32,0.62)$ & 2.78 & 0.249 \\
\hline $\begin{array}{l}\text { FBG } \\
(\mathrm{mmol} / \mathrm{L})\end{array}$ & $8.21 \pm 2.62$ & $7.13 \pm 2.32$ & $7.13 \pm 2.66$ & 15.71 & $<0.001$ \\
\hline $\begin{array}{l}\text { PBG } \\
(\mathrm{mmol} / \mathrm{L})\end{array}$ & $15.76 \pm 3.45$ & $15.49 \pm 3.76$ & $15.91 \pm 4.19$ & 0.90 & 0.410 \\
\hline
\end{tabular}




\begin{tabular}{|llllll|}
\hline HbA1C $(\%)$ & $9.07 \pm 2.18$ & $8.62 \pm 2.18$ & $8.48 \pm 2.08$ & 6.21 & 0.002 \\
\hline BMI $(\mathrm{kg} / \mathrm{m} 2)$ & $25.42 \pm 3.47$ & $25.43 \pm 4.29$ & $25.36 \pm 3.53$ & 0.04 & 0.965 \\
\hline SUDOSCAN & - & - & - & - & - \\
\hline $\begin{array}{l}\text { Hand ESC } \\
(\mu \mathrm{S})\end{array}$ & $70.97 \pm 13.34$ & $68.57 \pm 14.52$ & $65.25 \pm 20.12$ & 9.60 & $<0.001$ \\
\hline $\begin{array}{l}\text { Foot ESC } \\
(\mu \mathrm{S})\end{array}$ & $74.78 \pm 16.88$ & $73.04 \pm 18.27$ & $65.84 \pm 20.97$ & 19.48 & $<0.001$ \\
\hline HASYM $(\%)$ & $4.00(2.00,8.00)$ & $5.00(2.00,10.00)$ & $6.00(3.00,14.00)$ & 28.16 & $<0.001$ \\
\hline FASYM $(\%)$ & $2.00(1.00,4.00)$ & $2.00(1.00,6.25)$ & $3.00(1.00,10.00)$ & 20.83 & $<0.001$ \\
\hline
\end{tabular}

a SBP, systolic blood pressure, DBP, diastolic blood pressure, T-BIL, total bilirubin, D-BIL, direct bilirubin, BUN, blood urea nitrogen, $\mathrm{SCr}$, serum creatinine, eGFR, estimated glomerular filtration rate, UA, uric acid, Cys-C, cystatin C, TC, total cholesterol, TG, triglyceride, HDL, high density lipoprotein, LDL, low density lipoprotein, Hcy, homocysteine, FFA, free fat acid, FBG, fasting blood glucose, PBG, postprandial blood glucose, $\mathrm{HbA} 1 \mathrm{C}$, hemoglobin $\mathrm{A}_{1} \mathrm{C}, \mathrm{BMI}$, body mass index, ESC, electrochemical skin conductance, HASYM, asymmetry ratio in hands, FASYM, asymmetry ratio in feet.

${ }^{b}$ Normally distributed values in the table are presented as the means \pm SD, non-normally distributed values are presented as medians ( $25 \%$ and $75 \%$ interquartiles) and categorical variables are presented as frequencies (percentages). One way ANOVA for comparison of three samples with a normal distribution, Wilcoxon rank-sum test for abnormal distributions..X2-test for categorical variables.

Table 3 The association between clinical characteristics and metrics of SUDOSCAN. 


\begin{tabular}{|llllll|}
\hline & & Hand ESC & Foot ESC & HASYM & FASYM \\
\hline Age & $r$ & -0.189 & -0.198 & 0.153 & 0.155 \\
\hline Diabetes duration & $r$ & -0.173 & -0.183 & 0.103 & 0.152 \\
\hline SBP & $P$ & $<0.001$ & $<0.001$ & 0.002 & $<0.001$ \\
\hline & $r$ & -0.094 & -0.091 & 0.067 & 0.066 \\
\hline DBP & $P$ & 0.005 & 0.006 & 0.044 & 0.047 \\
\hline & $r$ & 0.033 & 0.002 & -0.019 & 0.026 \\
\hline eGFR & $P$ & 0.313 & 0.959 & 0.559 & 0.436 \\
\hline & $r$ & 0.106 & 0.198 & -0.178 & -0.159 \\
\hline HbA1C & $P$ & 0.001 & $<0.001$ & $<0.001$ & $<0.001$ \\
\hline & $r$ & -0.055 & 0.016 & -0.010 & -0.013 \\
\hline BMI & $P$ & 0.101 & 0.634 & 0.772 & 0.698 \\
\hline & $r$ & 0.085 & 0.059 & -0.009 & -0.064 \\
\hline Cys-C & $P$ & 0.010 & 0.073 & 0.776 & 0.051 \\
\hline & $r$ & -0.143 & -0.178 & 0.169 & 0.174 \\
\hline & $P$ & $<0.001$ & $<0.001$ & $<0.001$ & $<0.001$ \\
\hline
\end{tabular}

a SBP, systolic blood pressure, DBP, diastolic blood pressure, eGFR, estimated glomerular filtration rate, $\mathrm{HbA1C}$, hemoglobin A1C, BMI, body mass index, Cys-C, cystatin C ESC, electrochemical skin conductance, HASYM, asymmetry ratio in hands, FASYM, asymmetry ratio in feet.

Table 4 Multivariate regression linear analyses of association between serum Cys-C and parameters of SUDOSCAN. 


\begin{tabular}{|llllll|}
\hline & & $B$ & $S E$ & $\otimes$ & $P$ \\
\hline HESC & Cys-C & -14.657 & 3.737 & -0.276 & $<0.001$ \\
\hline FESC & Cys-C & -10.015 & 4.346 & -0.156 & 0.022 \\
\hline & Age & -0.415 & 0.200 & -0.267 & 0.039 \\
& Diabetes duration & -0.360 & 0.170 & -0.128 & 0.034 \\
\hline & BMI & 0.613 & 0.282 & 0.120 & 0.031 \\
\hline & HDL & 0.669 & 0.320 & 0.112 & 0.037 \\
\hline & e GFR & 0.426 & 0.202 & 0.360 & 0.036 \\
\hline & Cys-C & 4.023 & 2.219 & 0.125 & 0.071 \\
\hline FASYM & SBP & 0.192 & 0.047 & 0.309 & $<0.001$ \\
\hline & DBP & -0.179 & 0.075 & -0.177 & 0.017 \\
\hline & BMI & 2.757 & 1.906 & 0.100 & 0.149 \\
\hline & TC & -0.400 & 0.124 & -0.182 & 0.001 \\
\hline
\end{tabular}

${ }^{a}$ Cys-C, cystatin C, ESC, electrochemical skin conductance, HASYM, asymmetry ratio in hands, FASYM, asymmetry ratio in feet, eGFR, estimated glomerular filtration rate, BMI, body mass index, SBP, systolic blood pressure, DBP, diastolic blood pressure, TC, total cholesterol, B, Regression coefficient, SE, Standard error, $\beta$, Standardized coefficient.

${ }^{b}$ It was adjusted for age, diabetes duration, Cys-C, SBP, DBP, BMI, HbA1C, TC,HDL, FFA, Scr and eGFR.

\section{Figures}




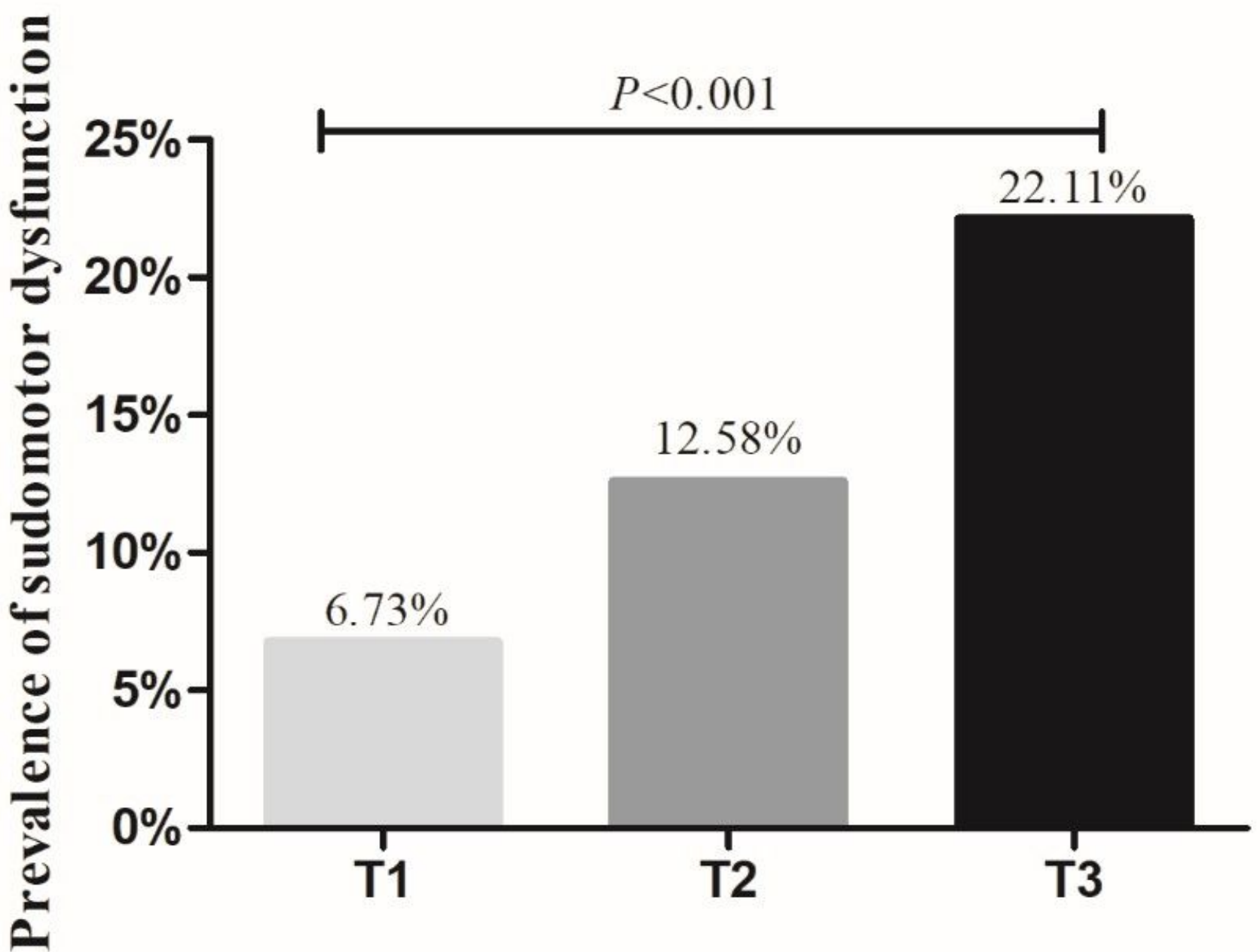

Figure 1

Prevalence of sudomotor dysfunction in different tertiles of serum Cys-C level. 